\title{
The development of mesenchymal stem cell therapy in the present, and the perspective of cell-free therapy in the future
}

\author{
Yusuke Watanabe', Atsunori Tsuchiya ${ }^{2}$, and Shuji Terai ${ }^{2}$ \\ ${ }^{1}$ Department of Preemptive Medicine for Digestive Disease and Healthy Active Life, School of Medicine, Niigata University, Niigata; \\ 2Division of Gastroenterology and Hepatology, Graduate School of Medical and Dental Sciences, Niigata University, Niigata, Japan
}

Cirrhosis is a chronic condition that can lead to liver failure. Currently, the viable option for decreasing mortality is liver transplantation. However, transplant surgery is highly invasive. Therefore, cell-based therapy has been developed as an alternative. Based on promising findings from preclinical research, some new trials have been registered. One of them was autologous bone marrow cell infusion therapy and found that ameliorating liver fibrosis activated liver regeneration. Now, majority of trials focus on low-immunogenicity mesenchymal stem cells (MSCs) appropriate for allogeneic administration. However, despite about 20 years of research, only a limited number of cell-based therapies have entered routine practice. Furthermore, potential shortcomings of cell-based therapy include a limit on the number of cells, which may be administered, as well as their failure to infiltrate target organs. On the other hand, these research show that MSCs act as "conducting cells" and regulate host cells including macrophages via extracellular vesicles (EVs) or exosome signals, leading to ameliorate liver fibrosis and promote regeneration. Therefore, the concept of cell-free therapy, which makes use of cell-derived EVs or exosomes, is attracting attention. Cell-free therapies may be safely administered in large doses and are able to infiltrate target organs. However, development of cell-free therapy exhibits its own set of challenges and such therapy may not be completely curative in the context of liver disease. This review describes the history of cell-based therapy research and recent advances in cell-free therapy, as well as discussing the need for more effective therapies. (Clin Mol Hepatol 2021;27:70-80)

Keywords: Cirrhosis; Extracellular vesicles; Exosomes; Mesenchymal stem cells; Macrophages

\begin{abstract}
Abbreviations:
ABMi, autologous bone marrow cell infusion; ACLF, acute-on-chronic liver failure; CSF, colony-stimulating factor; ECM, excessive extracellular matrix; EVs, extracellular vesicles; HGF, hepatocyte growth factor; HSCs, hepatic stellate cells; IL, interleukin; MELD, model for end-stage liver disease; MMPs, matrix metalloproteinases; MSCs, mesenchymal stem cells; OSM, oncostatin M; PGE2, prostaglandin E2; TSG, tumor necrosis factor-stimulated gen
\end{abstract}

\section{Corresponding author: Yusuke Watanabe}

Department of Preemptive Medicine for Digestive Disease and Healthy Active Life, School of Medicine, Niigata University, 1-757 Asahimachi-dori, Chuo-ku, Niigata 951-8510, Japan

Tel: +81-25-227-2207, Fax: +81-25-227-0776

E-mail:ywatanabe19840421@med.niigata-u.ac.jp

https://orcid.org/0000-0001-7869-0409 
Yusuke Watanabe, et al. A novel approach for cirrhosis patients

\section{INTRODUCTION}

Chronic liver disease and cirrhosis are life-threatening conditions — causing approximately 1-1.2 million deaths per yeararising in response to several etiologic factors including hepatitis virus infection, alcohol, nonalcoholic steatohepatitis, autoimmunity, and primary biliary cholangitis. ${ }^{1-3}$ In recent years, medical advances have significantly improved the prognosis of liver disease. ${ }^{4}$ Since the liver is highly regenerative, fibrosis can be at least partially ameliorated and liver function improved by eliminating the causative factor underlying dysfunction. However, once cirrhosis advances beyond a certain point, elimination of the causative factor will no longer facilitate regression of fibrosis, and cirrhosis may progress to carcinogenesis and/or liver failure. ${ }^{5-7}$ Therefore, the number of deaths attributable to cirrhosis remains high. ${ }^{8}$ Although liver transplantation is currently the only curative treatment for decompensated cirrhosis, ${ }^{9,10}$ significant limitations include a shortage of donors, high cost, highly invasive nature of the surgery, use of immunosuppressive agents to prevent graft rejection, and the potential for severe complications." Thus, cell-based therapy is of interest as an alternate and novel strategy to ameliorate liver fibrosis, reduce inflammation, and promote liver regeneration.

A good understanding of the mechanisms underlying liver fibrosis may contribute to therapeutically promoting its reversal. During liver injury, normally-quiescent hepatic stellate cells (HSCs) become activated by several cytokines and trophic factors, including transforming growth factor- $\beta$ and platelet-derived growth factor, leading to production of excessive extracellular matrix (ECM) constituents by activated myofibroblasts. Once the hepatotoxic stimulus is removed, the number of activated myofibroblasts regresses via apoptosis, senescence, and reversion to an inactive phenotype. Furthermore, matrix metalloproteinases (MMPs) are capable of ECM degradation. Thus, understanding the molecular mechanisms involved in HSC activation, ECM degradation, and myofibroblast involution or deactivation could facilitate manipulation of these processes and may lead to rational development of novel therapies for cirrhosis. ${ }^{12}$ Current therapeutic approaches to liver fibrosis are divided into five categories: 1) controlling primary liver disease, ${ }^{13,14}$ 2) targeting HSC receptor-ligand interactions and intracellular signaling, ${ }^{15-18} 3$ ) inhibiting fibrogenesis or matrix degradation, $\left.{ }^{19,20} 4\right)$ decreasing the number of activated $\mathrm{HSCS}^{21,22}$ and 5) cell-based therapy. Although cell-based therapy is mainly concerned with ameliorating liver fibrosis, it is also capable of producing other beneficial effects (including decreasing inflammation and promoting liver regeneration).
The ability of cell-based therapy to promote liver regeneration is also important, however. Regenerative factors including hepatocyte growth factor (HGF), oncostatin M (OSM), and Wnt3A which induce differentiation and influence phenotypic fate decisions of hepatic stem/progenitor cells - are secreted by macrophages. ${ }^{23,24}$ Furthermore, regeneration automatically occurs secondary to the anti-inflammatory and anti-fibrotic effects of cell-based therapy. It has been demonstrated that cell-based therapy, via dampening liver inflammation and ameliorating liver fibrosis, improves liver volume and function, including albumin synthesis and prothrombin time. ${ }^{25}$

At the inception of cell-based therapy during the period from 2003, first clinical study of autologous bone marrow cell infusion (ABMi) therapy was started in 2003, in which the patient's own bone marrow cells are harvested and re-injected. ${ }^{26-28}$ In this therapy, ABMi ameliorates liver fibrosis and improves liver function in cirrhotic patients (as indicated by increased serum albumin levels, decreased ascites, and decreased Child-Pugh scores) without inducing any major adverse events. ABMi also promotes a sequential activation of liver inherent regeneration in human livers. ${ }^{29-31}$ However, it is invasive, requiring general anesthesia in order to harvest $400 \mathrm{~mL}$ of bone marrow. Eligibility for $\mathrm{ABM} i$ requires a total bilirubin level of $\leq 3.0 \mathrm{mg} / \mathrm{dL}$ and a platelet count $\geq 50,000$ per $\mu \mathrm{L}$, representing criteria not met by all cirrhotic patients. Therefore, attention was shifted from bone marrow cells in general to specific stem cell subtypes. Research has focused largely on hematopoietic stem cells, ${ }^{32,33}$ mesenchymal stem cells (MSCs), ${ }^{34-36}$ and bone marrow-derived macrophages. ${ }^{37,38}$ In particular, MSCs and macrophages have been the mainstays of cell-based therapy, and their characteristics as well as relevant clinical trials will be described in the first half of this review.

More recently, it was noted that stem cell-derived extracellular vesicles (EVs) or exosomes also exhibit a therapeutic effect and may thus have potential for clinical application. ${ }^{39,40}$ While the development of cell-free therapy is still in its infancy, EV and exosome characteristics as well as relevant clinical trials will be described in the latter part of this review. Advantages and limitations of cell-based and cell-free therapies are discussed and future prospects regarding cirrhosis therapy are presented (Fig. 1).

\section{CELL-BASED THERAPY: CHARACTERISTICS OF MSCS AND MACROPHAGES}

Research into MSC-mediated cell-based therapy is conducted 


\section{CLINICAL and MOLECULAR}

\section{HEPATOLOGY}

Volume_27 Number_1 January 2021

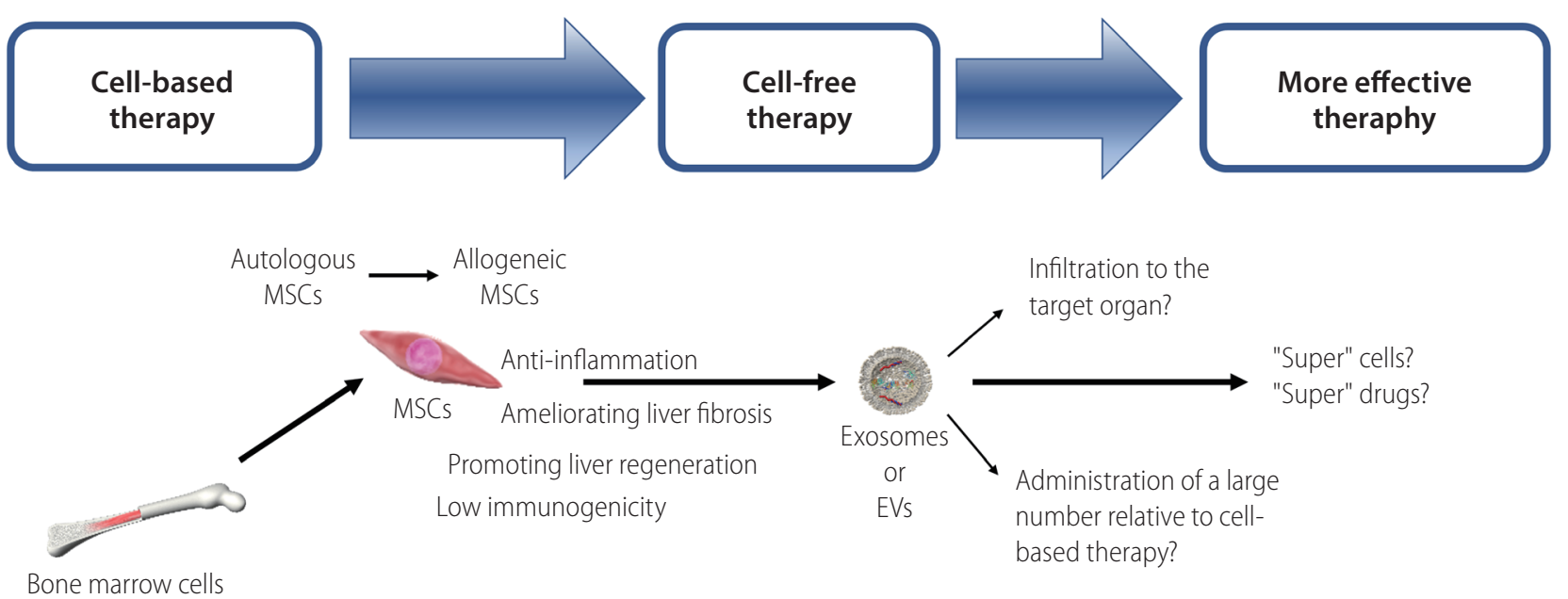

(ABMi)

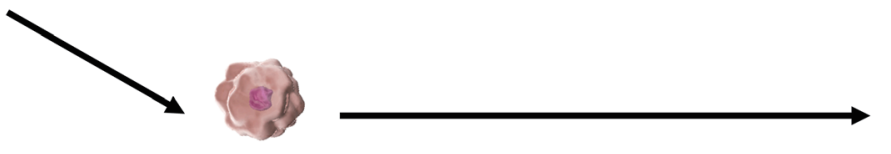

Drugs favoring M2

polarization of host

Macrophages

macrophages phenotype

Figure 1. Perspective: the flow from Cell-based therapy (ABMi, MSCs, and macropahges) to cell-free therapy (exosomes or EVs) to develop more effective therapy. MSCs, mesenchymal stem cells; ABMi, autologous bone marrow cell infusion therapy; EVs, extracellular vesicles.

globally. These cells are relatively easy to obtain, maintain, expand, and cryopreserve, all while maintaining their viability. For example, MSCs can be obtained not only from bone marrow but also from medical wastes such as adipose tissue, umbilical cord tissue, and dental pulp. ${ }^{41-51}$ Expression of common pluripotency markers (e.g., CD73, CD90, and CD105) is noted on MSCs, and they are able to differentiate into adipocytes, osteoblasts, and chondroblasts under the appropriate conditions. ${ }^{34,35}$

It appears that the major therapeutic (anti-inflammatory, antifibrotic, anti-oxidant, and angiogenic) effects of MSCs are due to their soluble products, including chemokines, cytokines, trophic factors, EVs, and exosomes. The most popular application of MSCs is decreasing inflammation. ${ }^{52}$ Various MSC products such as interleukin (IL)-10, tumor necrosis factor-stimulated gen (TSG)-6, nitric oxide, indoleamine2,3-dioxygenase, and prostaglandin E2 (PGE2) - are able to inhibit T-cell activation and expansion, induce regulatory $T$ lymphocyte, alter macrophage polarity towards less inflammatory phenotypes, and modulate natural killer cell, dendritic cell, and B-cell functions. ${ }^{53-56}$ By such mechanisms, MSCs are able to remotely influence the activities of many cellular effectors. ${ }^{57}$ Since recent studies suggest that MSC-conditioned medium (or exosomes present therein) are as effective as MSCs themselves, soluble factors are currently of great interest regarding their regenerative potential in the therapy of cirrho- sis. $^{7,34-36,57-60}$

Via soluble factors, MSCs may have therapeutic effects in cirrhosis even if they do not infiltrate the injured liver. Our group was the first to demonstrate in real time (using two-photon excitation microscopy) that peripheral intravenous administration of DsRed-labeled MSCs in a murine cirrhosis model resulted in the majority of MSCs being retained within the pulmonary vasculature, while only a few reached the liver. ${ }^{61}$ However, MSC-derived soluble factors (including EVs and exosomes) nonetheless exert a therapeutic effect on target organs (including the liver in context of cirrhosis), supporting the possibility of cell-free therapy.

Another important MSC characteristic is generally low immunogenicity. Since they express relatively low levels of major histocompatibility complex class I molecules and lack expression of major histocompatibility complex class II and co-stimulatory molecules (e.g., CD40, CD80, and CD86), they are not prone to triggering recipient immune responses ${ }^{57}$ and are thus suitable for allogeneic (as well as autologous) injection. Clinical studies suggest their therapeutic potential across disorders affecting a wide range of organs, including the liver, nerves, lungs, heart, and intestines.

Macrophages exhibit diverse phenotypes and high plasticity, ${ }^{37}$ including two major representative phenotypes: "classically activated" (M1) and "alternatively activated" (M2). During liver injury, pro-inflammatory M1 macrophages contribute to fibrosis via acti- 
Yusuke Watanabe, et al.

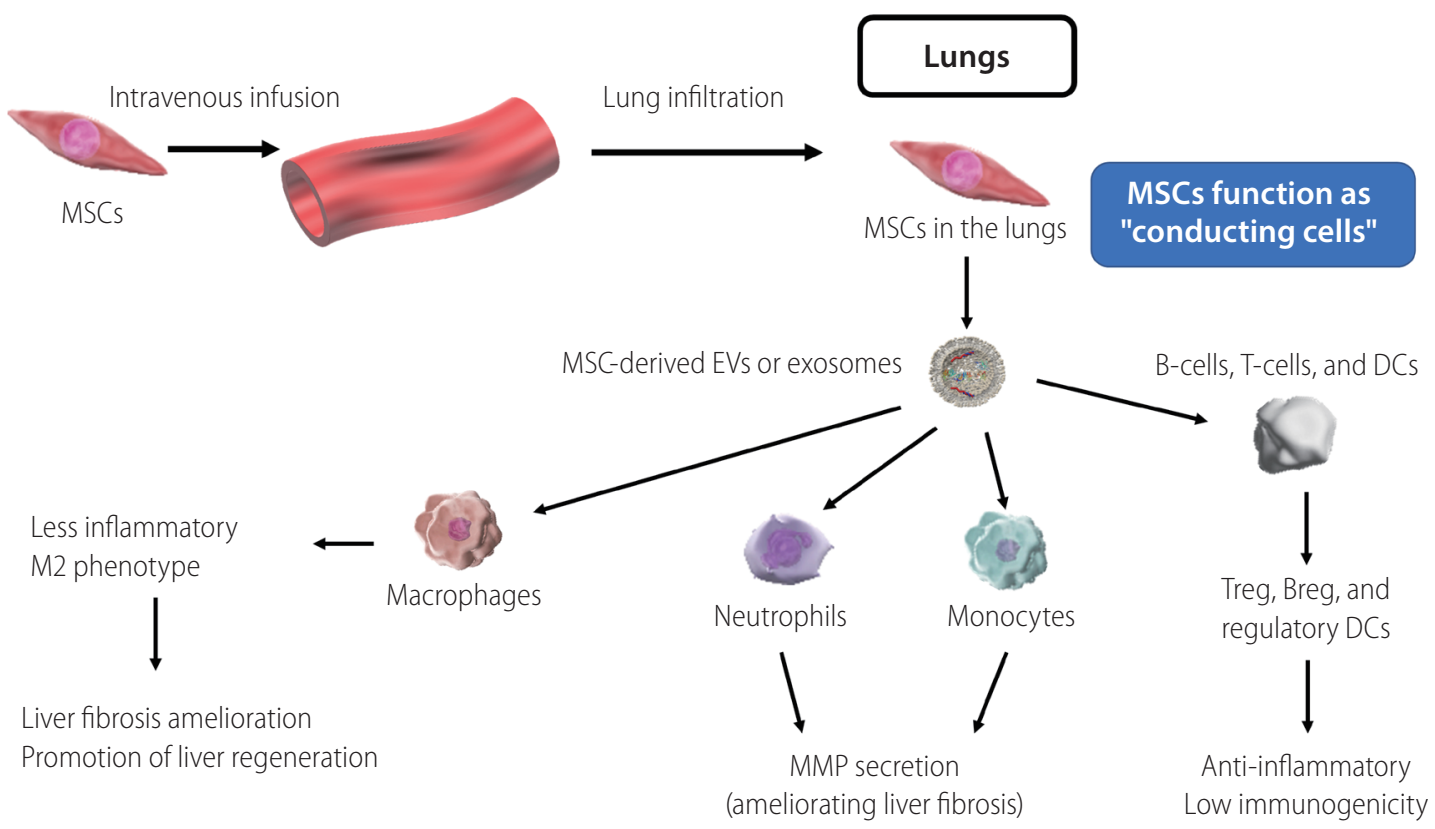

Figure 2. Overview of MSCs function as "conducting cells" to macrophages, neutrophils, monocytes, T-cells, B-cells, and DCs. MSCs, mesenchymal stem cells; EVs, extracellular vesicles; DCs, dendritic cells; MMP, matrix metalloproteinase.

vation of HSCS and myofibroblasts. In contrast, after stabilization of the liver injury process, M2 macrophages contribute to resolution of inflammation and reversal of fibrosis, the latter in part via MMPs. ${ }^{38}$ Furthermore, as mentioned, macrophages are able to influence differentiation and phenotypic fate decisions of hepatic stem/progenitor cells through production of HGF, OSM, and Wnt3A. ${ }^{23,24}$

Bone marrow-derived macrophages cultured in the presence of colony-stimulating factor (CSF)-1 induce regression of carbon tetrachloride-induced liver fibrosis in mice. ${ }^{62}$ We have previously reported that a large number of peripherally-administered green fluorescent protein-labeled macrophages are able to infiltrate the liver, where they help promote liver regeneration by phagocytizing hepatocytic debris. ${ }^{61}$ Although such findings demonstrate the therapeutic potential of macrophages in the context of cirrhosis, higher immunogenicity limits allogeneic macrophage administration. Instead, autologous macrophage administration can (e.g., via TSG-6, PGE2, and IL-13 ${ }^{63-65}$ ) help polarize host hepatic macrophages toward the less inflammatory/pro-resolving M2 phenotype, again supporting the potential of macrophages as novel cirrhotic therapeutic agents.

The same factors (TSG-6, PGE2, and IL-13) are also produced by MSCs, and contribute to host macrophages polarization in a similar manner. ${ }^{63-65}$ Furthermore, we have previously reported that
MSCs promote liver infiltration by host neutrophils and monocytes, which contribute to fibrosis amelioration via production of MMPs. ${ }^{61}$ These findings suggest that remote MSCs act as "conducting cells" (Fig. 2), via soluble factors including EVs or exosomes modulating macrophage, neutrophil, and monocyte functions in ways that ameliorate liver fibrosis and promote liver regeneration. ${ }^{53-57}$

\section{CELL-BASED THERAPY: CLINICAL TRIALS INVOLVING MSCS AND MACROPHAGES FOR LIVER DISEASE-RELEVANT INDICATIONS}

Although both MSCs and macrophages represent promising candidates for use in cell-based therapy, a majority of current clinical trials focus on MSCs. According to ClinicalTrials.gov, over 1,100 MSC-based clinical trials have been registered across a variety of disciplines, including 51 that began investigating cell-based therapy for liver disease after 2006. Thereafter, the number of cell-based therapy clinical trial registrations (incorporating cell sources including bone marrow, adipose tissue, umbilical cord tissue, and dental pulp, and routes of administration including peripheral venous and arterial injection ${ }^{47-49,66-69}$ ) gradually increased to a peak as the approach gained recognition. In 2017, we our- 
selves registered a phase I and II clinical trial (first clinical trial involving MSCs in Japan) focused on adipose-derived MSC infusion in cirrhosis patients (NCT: 03254758). Our clinical trial involving allogeneic MSCs (without using immunosuppressive agents) are proceeding into phase II after finishing phase I. However, the number of clinical trials has been declining since 2017 (Fig. 3). This trend is likely due to a decline in novelty, slow progress in translating preclinical research to clinical interventions, and a tendency of shift in focus toward cell-free therapy incorporating EVs

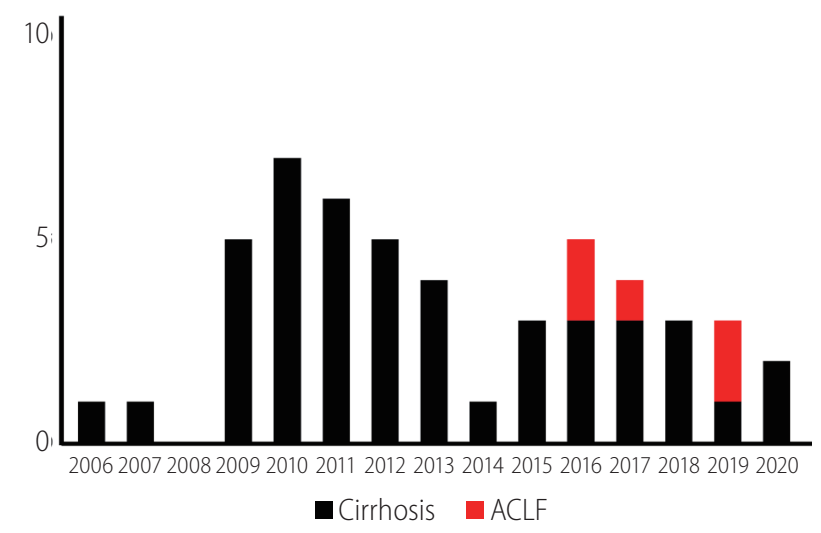

Figure 3. Number of clinical trials involving mesenchymal stem cells. Black bars represent trials focused on cirrhosis in general, while stacked red bars represent trials focused specifically on acute-on-chronic liver failure (ACLF).

\section{or exosomes.}

Furthermore, to describe recent trends in MSC-oriented clinical trials, we evaluated studies (12 studies) which began or will begin after January 2017 according to ClinicalTrials.gov (Table 1). The majority of these were registered in Asian countries. Studies vary in cell source, autologous versus allogeneic nature, and etiology of cirrhosis. However, in most studies (11 of 12) MSC administration is via peripheral injection of approximately $1 \times 10^{6} \mathrm{MSC} / \mathrm{kg}$ per dose. Studies focus not only on cirrhosis, but also on acute-onchronic liver failure (ACLF). Since MSCs exhibit a stronger therapeutic effect during inflammation, they may be particularly effective in the context of ACLF. Altogether, many MSC-oriented clinical trials have not yet progressed beyond phase I or II.

Regarding macrophage-oriented cell-based therapy, clinical trials further elucidating macrophage characteristics have also been reported by Edinburgh University. The first-in-human phase 1 dose-escalation trial of intravenous autologous macrophage therapy incorporated nine adults with cirrhosis and model for end-stage liver disease (MELD) scores of $10-16^{70}$ In this trial, macrophages were produced from patient mononuclear cell leukapheresis-derived monocytes cultured in the presence of CSF-1. Despite macrophage potential for immunogenicity, no transfusion reactions, dose-limiting toxicities, or macrophage activation syndromes were reported, and all participants remained alive and transplant-free at 1 year. Furthermore, after 1 year, MELD scores

Table 1. Recent clinical trials involving MSCS

\begin{tabular}{|c|c|c|c|c|c|c|c|c|}
\hline No. & Country & Cell origin & $\begin{array}{l}\text { Autologous/ } \\
\text { allogeneic }\end{array}$ & $\begin{array}{l}\text { Administration } \\
\text { route }\end{array}$ & Number of cells infused & Etiology & $\begin{array}{l}\text { No. of } \\
\text { patients }\end{array}$ & $\begin{array}{c}\text { Trial } \\
\text { (phase) }\end{array}$ \\
\hline 1 & China & N.A & N.A. & IV from peripheral & $1.0 \times 10^{5}-10^{6}$ cells $/ \mathrm{kg}, 3$ times & ACLF & 200 & N.A. \\
\hline 2 & China & Umbilical cord & Allogeneic & IV from peripheral & $1.5 \times 10^{6}$ cells $/ \mathrm{kg}, 4$ times & Cirrhosis & 252 & Phase 2 \\
\hline 3 & China & N.A. & N.A. & IV from peripheral & $1.0 \times 10^{6}$ cells $/ \mathrm{kg}, 3$ times & Cirrhosis & 200 & N.A. \\
\hline 4 & Japan & Adipose tissue & Allogeneic & IV from peripheral & N.A. & Cirrhosis & 15 & Phase $1 / 2$ \\
\hline 5 & China & N.A. & N.A. & IV from peripheral & N.A. & Cirrhosis & 30 & Phase $1 / 2$ \\
\hline 6 & China & N.A. & N.A. & IV from peripheral & $1.0 \times 10^{5}-10^{6}$ cells $/ \mathrm{kg}, 4$ times & ACLF & 45 & Phase $1 / 2$ \\
\hline 7 & Germany & $\begin{array}{l}\text { Skin-derived } \\
\text { ABCD5 + cells }\end{array}$ & Allogeneic & IV from peripheral & $2.0 \times 10^{6}$ cells $/ \mathrm{kg}, 3$ times & ACLF & 18 & Phase $1 / 2$ \\
\hline 8 & China & Umbilical cord & N.A. & IV from peripheral & $1.0 \times 10^{6}$ cells $/ \mathrm{kg}, 3$ times & Cirrhosis & 200 & Phase 2 \\
\hline 9 & Taiwan & Adipose tissue & Allogeneic & IV from peripheral & $0.5-2.0 \times 10^{6}$ cells $/ \mathrm{kg}$ & ACLF & 20 & Phase 1 \\
\hline 10 & Singapore & Bone marrow & Autologous & IV from peripheral & $0.5-1.0 \times 10^{6}$ cells $/ \mathrm{kg}$ & Cirrhosis & 20 & Phase $1 / 2$ \\
\hline 11 & India & N.A. & Autologous & IA from hepatic & N.A. & Cirrhosis & 5 & Phase 4 \\
\hline 12 & Indonesia & Umbilical cord & Allogeneic & IV from peripheral & $1.0 \times 10^{8}$ cells & Cirrhosis & 12 & Phase $1 / 2$ \\
\hline
\end{tabular}

The trial of No. 4 is ours.

MSCS, mesenchymal stem cells; N.A., not applicable; IV, intravenously injection; ACLF, acute-on-chronic liver failure; IA, intra-arterial injection. 
had decreased in seven out of nine patients, and a liver fibrosis marker had decreased in five out of nine patients. Because macrophages are also an essential component in liver regeneration, acceleration of research in this area is anticipated.

\section{CELL-BASED THERAPY: ADVANTAGES AND LIMITATIONS}

As alluded to, cell-based therapy has many advantages. Especially so for MSCs, cells are relatively easy to obtain, culture, expand, characterize for quality control, count, and assess for viability. ${ }^{41-51}$ In addition, since MSC therapeutic effects are enhanced during inflammation, preconditioning can strengthen their therapeutic effects. Finally, numerous avenues of cell modification are possible. Despite administered cells becoming trapped in the pulmonary vasculature, ${ }^{61}$ existing clinical trials report few major adverse events, including pulmonary embolism. ${ }^{47}$ Although administered macrophages are autologous due to immunogenicity-based constraints, they are now obtainable via less invasive methods. ${ }^{70}$ Moreover, the ability to elicit and influence host macrophage development locally within the liver (i.e., without the need for exogenous administration) would be an even more attractive possibility.

However, cell-based therapy also has certain limitations. For example, concerns regarding the risk of pulmonary embolism continue to limit dosage, and administered cells do not access the target organ. Although cells do continue to exert a therapeutic effect remotely, a therapeutic agent acting directly within damaged sites would be preferable. Furthermore, given the propensity of stem cells to differentiate, it is difficult to maintain a stable phenotype during long-term culture. Therefore, commercialization would necessitate rigorous quality control. Such concerns have driven the search for alternate therapeutic strategies and cell-free therapy may overcome some of the limitations associated with cell-based therapy.

\section{CELL-FREE THERAPY IN THE FUTURE: CHARAC- TERISTICS OF EVS AND EXOSOMES}

The generic term for membrane-bound particles naturally released by cells is EVs; exosomes are a subtype of these. ${ }^{71}$ Once believed to be "trash bags" for cellular debris, EVs and exosomes are now being explored for their potential as next-generation diagnostic and therapeutic tools. ${ }^{72}$ The heterogeneous group of EVs encompasses a variety of particles of different sizes with varying contents. Exosomes, specifically, are 40-100 nm in diameter corresponding to a density of $1.13-1.19 \mathrm{~g} / \mathrm{mL}$ in sucrose solution - and can be sedimented by ultracentrifugation at 100,000 $x g^{72}$ They contain a mixture of proteins, messenger RNA, transfer RNA, micro RNA, genomic DNA, and complementary DNA. ${ }^{73}$

Like stem cells, exosomes exhibit many biological activities and have shown therapeutic potential in several organ system and disease contexts. For example, exosomes may: protect against cisplatin-induced renal oxidative stress and renal cell apoptosis, ${ }^{74}$ enhance myocardial viability and prevent adverse remodeling after ischemic injury, ${ }^{75}$ promote angiogenesis in the setting of myocardial infarction, ${ }^{76,77}$ protect the intestines from enterocolitis, ${ }^{78}$ improve hypoxia-induced pulmonary hypertension, ${ }^{79-81}$ and promote functional recovery after stroke. ${ }^{82,83}$ At least in part, such therapeutic effects of exosomes are attributable to their ability to induce angiogenesis as well as regeneration and proliferation of epithelia. ${ }^{84-86}$ Furthermore, exosomes exhibit immunomodulatory (largely anti-inflammatory) effects, some of which — specifically down-regulation of interferon- $\gamma$ secretion and T-cell polarity alteration - are able to stabilize skin graft survival. ${ }^{87,88}$ Many studies have found that the efficacy of exosomes nearly matches that of cell-based therapy, indicating that exosomes have potential as next-generation (i.e., cell-free) therapy. ${ }^{76,89-91}$

Specifically regarding liver disease, we previously reported that MSCs exhibit remote therapeutic effects, ${ }^{61}$ a phenomenon attributable to EVs or exosomes. Furthermore, exosomes are reported to ameliorate liver fibrosis in cirrhotic mice, ${ }^{92-97}$ promote liver regeneration and hepatocyte proliferation (via up-regulation of $\mathrm{BCl}$ $\mathrm{xl}$ protein) in a murine liver injury model, ${ }^{92}$ alleviate acute liver failure by dampening macrophage NLRP3 inflammasome activity (additionally suggesting their potential utility during acute liver in-

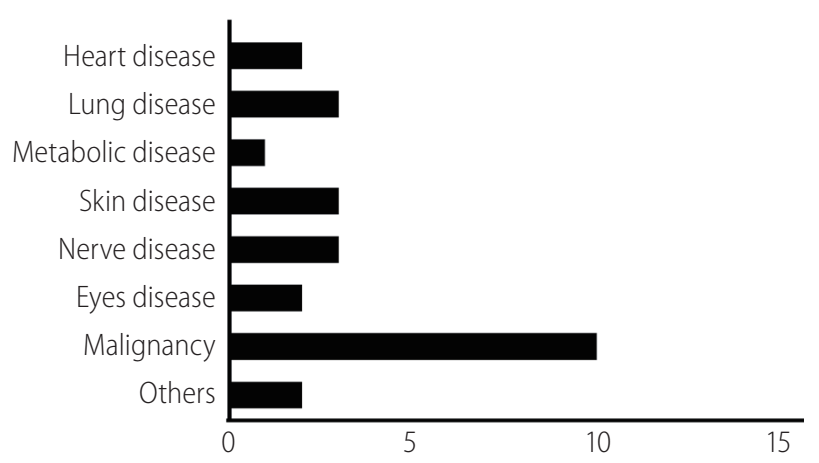

Figure 4. Number of clinical trials involving extracellular vesicles or exosomes. 
jury) ${ }_{1}{ }^{94}$ and improve survival after D-galactosamine- and tumor necrosis factor-a-induced hepatic failure in mice. ${ }^{95}$ The latter study also demonstrated that peripherally-administered fluorescently-labeled exosomes were preferentially detectable at high levels in damaged (relative to normal) liver at 6 hours post-administration. ${ }^{95}$ Relatedly, it has been shown that intravenously injected exosomes which infiltrate the liver are internalized by hepatic macrophages. ${ }^{98}$ Such results suggest that exosomes do not infiltrate the lungs but accumulate in target organs (e.g., sites of liver damage).

\section{CELL-FREE THERAPY IN THE FUTURE: CLINICAL TRIALS INVOLVING EVS AND EXOSOMES}

In order to describe relevant recent clinical research trends, we identified EV- or exosome-oriented clinical trials registered on ClinicalTrials.gov. Results included trials focused on both therapeutic applications of exosomes and cancer diagnostic exosome analysis. Excluding observational studies, 26 exosome-oriented therapeutic clinical trials were registered. Of these, 10 focused on cancer diagnostics; three each focused on pulmonary disease, neurological disease, and dermatological disease; two each focused on heart disease, ophthalmological disease, and multiple organ failure, and one focused on metabolic disease (Fig. 4). To the best of our knowledge, no EV- or exosome-oriented clinical trial focused on liver disease was registered at this time. The small number of clinical studies (and the absence of any studies focused on liver disease) is likely attributable to the absence of a simple, standardized method for the production of large quantities of EVs or exosomes; an inadequate understanding of the mechanisms of action of cell-free therapy; and insufficient data regarding the behavior of administered EVs or exosomes in liver disease. However, given the therapeutic potential of EVs and exosomes in liver disease, the number of relevant clinical trials is expected to increase gradually.

\section{CELL-FREE THERAPY IN THE FUTURE: ADVAN- TAGES AND LIMITATIONS}

Given the small diameter of EVs and exosomes, the associated risk of thrombosis after intravenous administration is considered much lower relative to that posed by stem cells (e.g., MSCs). If it is confirmed that cell-free therapeutic effects are dose-dependent, the ability to administer large quantities of EVs or exosomes will be a significant advantage. In addition, the ability of EVs or exosomes to infiltrate damaged target organs directly may result in greater efficacy relative to that of distant stem cells. Some studies have even examined the application of EVs or exosomes to targeted drug delivery. ${ }^{99}$

However, even though cell-free therapy overcomes many of the limitations associated with cell-based therapy, the former, too, has its limitations. As mentioned, no standardized method yet exists for the production of large quantities of EVs or exosomes. Furthermore, it is unclear whether using isolated EVs or exosomes excludes an important factor underlying cell-based therapy associated therapeutic effects. Finally, due to the rapid pace of cellfree therapy development, relevant medico-legal norms surrounding cell-free therapy are not yet sufficiently established. Clearly, the field of cell-free therapy is still in its infancy, requiring significant further research.

\section{CONCLUSIONS AND FUTURE PROSPECTS}

Cell-based therapy has been developed as an alternative to liver transplantation, but has not yet progressed beyond early-phase clinical trials. Nonetheless, cell-free therapy — which overcomes some of the limitations associated with cell-based therapy- is already under development as a next-generation therapeutic technology, and clinical trials relevant to liver disease are expected to begin in the near future. Cell-free therapy has its own limitations, however. One promising strategy to identify novel and effective curative therapies may be elucidation the mechanisms underlying EV and exosome biological activities, in order to isolate the responsible molecules for direct administration to patients. In conclusion, many challenges remain in developing curative therapies for cirrhosis, but further research into cell-based and cell-free therapies will likely be of significant benefit.

\section{Authors' contribution}

YW reviewed and interpreted prior literature and case studies/ clinical trials. YW, AT, and ST contributed to manuscript preparation and review prior to submission.

\section{Acknowledgements}

This study was supported by the Research Program on Hepatitis from Japan Agency for Medical Research and Development (AMED) (20fk0210070h0001). 


\section{Conflicts of Interest}

The authors have no conflicts to disclose.

\section{REFERENCES}

1. Tsochatzis EA, Bosch J, Burroughs AK. Liver cirrhosis. Lancet 2014;383:1749-1761.

2. Asrani SK, Devarbhavi H, Eaton J, Kamath PS. Burden of liver diseases in the world. J Hepatol 2019;70:151-171.

3. Tateishi R, Uchino K, Fujiwara N, Takehara T, Okanoue T, Seike M, et al. A nationwide survey on non- $B$, non- $C$ hepatocellular carcinoma in Japan: 2011-2015 update. J Gastroenterol 2019;54:367-376.

4. Tang LSY, Covert E, Wilson E, Kottilil S. Chronic hepatitis B infection: a review. JAMA 2018;319:1802-1813.

5. Jang JW, Choi JY, Kim YS, Woo HY, Choi SK, Lee CH, et al. Longterm effect of antiviral therapy on disease course after decompensation in patients with hepatitis B virus-related cirrhosis. Hepatology 2015;61:1809-1820.

6. Curry MP, O'Leary JG, Bzowej N, Muir AJ, Korenblat KM, Fenkel JM, et al. Sofosbuvir and velpatasvir for HCV in patients with decompensated cirrhosis. N Engl J Med 2015;373:2618-2628.

7. Terai S, Tsuchiya A. Status of and candidates for cell therapy in liver cirrhosis: overcoming the "point of no return" in advanced liver cirrhosis. J Gastroenterol 2017;52:129-140.

8. Mokdad AA, Lopez AD, Shahraz S, Lozano R, Mokdad AH, Stanaway J, et al. Liver cirrhosis mortality in 187 countries between 1980 and 2010: a systematic analysis. BMC Med 2014;12:145.

9. Toniutto P, Bitetto D, Fornasiere E, Fumolo E. Challenges and future developments in liver transplantation. Minerva Gastroenterol Dietol 2019;65:136-152

10. Forbes SJ, Gupta S, Dhawan A. Cell therapy for liver disease: from liver transplantation to cell factory. J Hepatol 2015;62(1 Suppl):S157S169.

11. Merion RM, Schaubel DE, Dykstra DM, Freeman RB, Port FK, Wolfe RA. The survival benefit of liver transplantation. Am J Transplant 2005:5:307-313

12. Schuppan D, Ashfaq-Khan M, Yang AT, Kim YO. Liver fibrosis: direct antifibrotic agents and targeted therapies. Matrix Biol 2018;6869:435-451.

13. Sanyal AJ, Chalasani N, Kowdley KV, McCullough A, Diehl AM, Bass $N M$, et al. Pioglitazone, vitamin $E$, or placebo for nonalcoholic steatohepatitis. N Engl J Med 2010;362:1675-1685.

14. Traber PG, Chou H, Zomer E, Hong F, Klyosov A, Fiel MI, et al. Regression of fibrosis and reversal of cirrhosis in rats by galectin inhibitors in thioacetamide-induced liver disease. PLoS One 2013:8:e75361.
15. Granzow M, Schierwagen R, Klein S, Kowallick B, Huss S, Linhart M, et al. Angiotensin-II type 1 receptor-mediated Janus kinase 2 activation induces liver fibrosis. Hepatology 2014;60:334-348.

16. Rozenfeld R, Gupta A, Gagnidze K, Lim MP, Gomes I, Lee-Ramos $D$, et al. AT1R-CB1R heteromerization reveals a new mechanism for the pathogenic properties of angiotensin II. EMBO J 2011;30:23502363.

17. Ueno $T$, Nakamura A, Nakayama $H$, Otabe $S$, Yuan X, Fukutani T, et al. Adiponectin suppresses endoplasmic reticulum stress in nonalcoholic steatohepatitis. Exp Ther Med 2011;2:1035-1040.

18. Moreno M, Chaves JF, Sancho-Bru P, Ramalho F, Ramalho LN, Mansego ML, et al. Ghrelin attenuates hepatocellular injury and liver fibrogenesis in rodents and influences fibrosis progression in humans. Hepatology 2010;51:974-985.

19. Puthawala K, Hadjiangelis N, Jacoby SC, Bayongan E, Zhao Z, Yang $Z$, et al. Inhibition of integrin alpha(v)beta6, an activator of latent transforming growth factor-beta, prevents radiation-induced lung fibrosis. Am J Respir Crit Care Med 2008;177:82-90.

20. Henderson NC, Arnold TD, Katamura Y, Giacomini MM, Rodriguez $J D, M c C a r t y ~ J H$, et al. Targeting of av integrin identifies a core molecular pathway that regulates fibrosis in several organs. Nat Med 2013;19:1617-1624.

21. Gao B, Radaeva S, Park O. Liver natural killer and natural killer T cells: immunobiology and emerging roles in liver diseases. J Leukoc Biol 2009;86:513-528.

22. Chakraborty JB, Mann DA. NF-kappaB signalling: embracing complexity to achieve translation. J Hepatol 2010;52:285-291.

23. Kamiya A, Kinoshita T, Ito Y, Matsui T, Morikawa Y, Senba E, et al. Fetal liver development requires a paracrine action of oncostatin $M$ through the gp130 signal transducer. EMBO J 1999;18:2127-2136.

24. Boulter L, Govaere O, Bird TG, Radulescu S, Ramachandran P, Pellicoro A, et al. Macrophage-derived Wnt opposes Notch signaling to specify hepatic progenitor cell fate in chronic liver disease. Nat Med 2012;18:572-579.

25. Peng L, Xie DY, Lin BL, Liu J, Zhu HP, Xie C, et al. Autologous bone marrow mesenchymal stem cell transplantation in liver failure patients caused by hepatitis B: short-term and long-term outcomes. Hepatology 2011;54:820-828.

26. Sakaida I, Terai S, Yamamoto N, Aoyama K, Ishikawa T, Nishina H, et al. Transplantation of bone marrow cells reduces $\mathrm{CCl} 4$-induced liver fibrosis in mice. Hepatology 2004;40:1304-1311.

27. Terai S, Ishikawa T, Omori K, Aoyama K, Marumoto Y, Urata Y, et al. Improved liver function in patients with liver cirrhosis after autologous bone marrow cell infusion therapy. Stem Cells 2006;24:22922298.

28. Terai S, Sakaida I. Current status of autologous bone marrow cell infusion therapy for liver cirrhosis patients. Hepatol Res 2008;38 Suppl 1:S72-S75. 
29. Terai S, Tanimoto H, Maeda M, Zaitsu J, Hisanaga T, Iwamoto T, et al. Timeline for development of autologous bone marrow infusion (ABMi) therapy and perspective for future stem cell therapy. J Gastroenterol 2012;47:491-497.

30. Saito T, Okumoto K, Haga H, Nishise Y, Ishii R, Sato C, et al. Potential therapeutic application of intravenous autologous bone marrow infusion in patients with alcoholic liver cirrhosis. Stem Cells Dev 2011;20:1503-1510.

31. Kim JK, Park YN, Kim JS, Park MS, Paik YH, Seok JY, et al. Autologous bone marrow infusion activates the progenitor cell compartment in patients with advanced liver cirrhosis. Cell Transplant 2010;19:1237-1246.

32. Nakamura T, Torimura T, Sakamoto M, Hashimoto O, Taniguchi E, Inoue $\mathrm{K}$, et al. Significance and therapeutic potential of endothelial progenitor cell transplantation in a cirrhotic liver rat model. Gastroenterology 2007;133:91-107.e1.

33. Salama H, Zekri AR, Zern M, Bahnassy A, Loutfy S, Shalaby S, et al. Autologous hematopoietic stem cell transplantation in 48 patients with end-stage chronic liver diseases. Cell Transplant 2010;19:14751486.

34. Wang Y, Chen X, Cao W, Shi Y. Plasticity of mesenchymal stem cells in immunomodulation: pathological and therapeutic implications. Nat Immunol 2014;15:1009-1016.

35. Zhou BO, Yue R, Murphy MM, Peyer JG, Morrison SJ. Leptin-receptor-expressing mesenchymal stromal cells represent the main source of bone formed by adult bone marrow. Cell Stem Cell 2014;15:154168.

36. Prockop DJ, Oh JY. Mesenchymal stem/stromal cells (MSCs): role as guardians of inflammation. Mol Ther 2012;20:14-20.

37. Sica A, Mantovani A. Macrophage plasticity and polarization: in vivo veritas. J Clin Invest 2012;122:787-795.

38. Duffield JS, Forbes SJ, Constandinou CM, Clay S, Partolina M, Vuthoori $S$, et al. Selective depletion of macrophages reveals distinct, opposing roles during liver injury and repair. J Clin Invest 2005;115:56-65.

39. Bruno S, Grange C, Collino F, Deregibus MC, Cantaluppi V, Biancone $L$, et al. Microvesicles derived from mesenchymal stem cells enhance survival in a lethal model of acute kidney injury. PLoS One 2012;7:e33115.

40. Parekkadan $B$, van Poll D, Suganuma K, Carter EA, Berthiaume $F$, Tilles AW, et al. Mesenchymal stem cell-derived molecules reverse fulminant hepatic failure. PLoS One 2007;2:e941.

41. Iwamoto T, Terai S, Hisanaga T, Takami T, Yamamoto N, Watanabe S, et al. Bone-marrow-derived cells cultured in serum-free medium reduce liver fibrosis and improve liver function in carbon-tetrachloridetreated cirrhotic mice. Cell Tissue Res 2013;351:487-495.

42. Tanimoto H, Terai S, Taro T, Murata Y, Fujisawa K, Yamamoto N, et al. Improvement of liver fibrosis by infusion of cultured cells derived from human bone marrow. Cell Tissue Res 2013;354:717-728.

43. Terai S, Takami T, Yamamoto N, Fujisawa K, Ishikawa T, Urata Y, et al. Status and prospects of liver cirrhosis treatment by using bone marrow-derived cells and mesenchymal cells. Tissue Eng Part B Rev 2014;20:206-210.

44. Banas A, Teratani T, Yamamoto Y, Tokuhara M, Takeshita F, Quinn G, et al. Adipose tissue-derived mesenchymal stem cells as a source of human hepatocytes. Hepatology 2007;46:219-228.

45. Seki A, Sakai Y, Komura T, Nasti A, Yoshida K, Higashimoto M, et al. Adipose tissue-derived stem cells as a regenerative therapy for a mouse steatohepatitis-induced cirrhosis model. Hepatology 2013;58:1133-1142.

46. Shiratsuki S, Terai S, Murata Y, Takami T, Yamamoto N, Fujisawa K, et al. Enhanced survival of mice infused with bone marrow-derived as compared with adipose-derived mesenchymal stem cells. Hepatol Res 2015;45:1353-1359.

47. Shi M, Zhang Z, Xu R, Lin H, Fu J, Zou Z, et al. Human mesenchymal stem cell transfusion is safe and improves liver function in acute-onchronic liver failure patients. Stem Cells Transl Med 2012;1:725-731.

48. Zhang Z, Lin H, Shi M, Xu R, Fu J, Lv J, et al. Human umbilical cord mesenchymal stem cells improve liver function and ascites in decompensated liver cirrhosis patients. J Gastroenterol Hepatol 2012;27 Suppl 2:112-120.

49. Wang L, Li J, Liu H, Li Y, Fu J, Sun Y, et al. Pilot study of umbilical cord-derived mesenchymal stem cell transfusion in patients with primary biliary cirrhosis. J Gastroenterol Hepatol 2013;28 Suppl 1:8592.

50. Wang L, Han Q, Chen H, Wang K, Shan GL, Kong F, et al. Allogeneic bone marrow mesenchymal stem cell transplantation in patients with UDCA-resistant primary biliary cirrhosis. Stem Cells Dev 2014;23:2482-2489.

51. Sharpe PT. Dental mesenchymal stem cells. Development 2016;143: 2273-2280.

52. Ikarashi S, Tsuchiya A, Kawata Y, Kojima Y, Watanabe T, Takeuchi $S$, et al. Effects of human adipose tissue-derived and umbilical cord tissue-derived mesenchymal stem cells in a dextran sulfate sodiuminduced mouse model. Biores Open access 2019;8:185-199.

53. Kawata Y, Tsuchiya A, Seino S, Watanabe Y, Kojima Y, Ikarashi S, et al. Early injection of human adipose tissue-derived mesenchymal stem cell after inflammation ameliorates dextran sulfate sodiuminduced colitis in mice through the induction of M2 macrophages and regulatory T cells. Cell Tissue Res 2019;376:257-271.

54. Volarevic V, Gazdic M, Simovic Markovic B, Jovicic N, Djonov V, Arsenijevic N. Mesenchymal stem cell-derived factors: immuno-modulatory effects and therapeutic potential. Biofactors 2017;43:633644.

55. Ma OK, Chan KH. Immunomodulation by mesenchymal stem cells: interplay between mesenchymal stem cells and regulatory lympho- 
Yusuke Watanabe, et al. A novel approach for cirrhosis patients

cytes. World J Stem Cells 2016;8:268-278.

56. Pinheiro D, Dias I, Ribeiro Silva K, Stumbo AC, Thole A, Cortez E, et al. Mechanisms underlying cell therapy in liver fibrosis: an overview. Cells 2019;8:1339.

57. Tsuchiya A, Takeuchi S, Watanabe T, Yoshida T, Nojiri S, Ogawa M, et al. Mesenchymal stem cell therapies for liver cirrhosis: MSCs as "conducting cells" for improvement of liver fibrosis and regeneration. Inflamm Regen 2019;39:18.

58. Tsuchiya A, Kojima Y, Ikarashi S, Seino S, Watanabe Y, Kawata Y, et al. Clinical trials using mesenchymal stem cells in liver diseases and inflammatory bowel diseases. Inflamm Regen 2017;37:16.

59. Kojima Y, Tsuchiya A, Ogawa M, Nojiri S, Takeuchi S, Watanabe T, et al. Mesenchymal stem cells cultured under hypoxic conditions had a greater therapeutic effect on mice with liver cirrhosis compared to those cultured under normal oxygen conditions. Regen Ther 2019;11:269-281.

60. Watanabe T, Tsuchiya A, Takeuchi S, Nojiri S, Yoshida T, Ogawa M, et al. Development of a non-alcoholic steatohepatitis model with rapid accumulation of fibrosis, and its treatment using mesenchymal stem cells and their small extracellular vesicles. Regen Ther 2020;14:252-261

61. Watanabe Y, Tsuchiya A, Seino S, Kawata Y, Kojima Y, Ikarashi S, et al. Mesenchymal stem cells and induced bone marrow-derived macrophages synergistically improve liver fibrosis in mice. Stem Cells Transl Med 2019:8:271-284.

62. Thomas JA, Pope C, Wojtacha D, Robson AJ, Gordon-Walker TT, Hartland $S$, et al. Macrophage therapy for murine liver fibrosis recruits host effector cells improving fibrosis, regeneration, and function. Hepatology 2011;53:2003-2015.

63. Kim J, Hematti P. Mesenchymal stem cell-educated macrophages: a novel type of alternatively activated macrophages. Exp Hematol 2009:37:1445-1453.

64. Eggenhofer $E$, Hoogduijn MJ. Mesenchymal stem cell-educated macrophages. Transplant Res 2012;1:12.

65. Maggini J, Mirkin G, Bognanni I, Holmberg J, Piazzón IM, Nepomnaschy I, et al. Mouse bone marrow-derived mesenchymal stromal cells turn activated macrophages into a regulatory-like profile. PLOS One 2010;5:e9252.

66. Kharaziha P, Hellström PM, Noorinayer B, Farzaneh F, Aghajani K, Jafari $F$, et al. Improvement of liver function in liver cirrhosis patients after autologous mesenchymal stem cell injection: a phase I-II clinical trial. Eur J Gastroenterol Hepatol 2009;21:1199-1205.

67. Jang YO, Kim YJ, Baik SK, Kim MY, Eom YW, Cho MY, et al. Histological improvement following administration of autologous bone marrow-derived mesenchymal stem cells for alcoholic cirrhosis: a pilot study. Liver Int 2014;34:33-41.

68. Suk KT, Yoon JH, Kim MY, Kim CW, Kim JK, Park H, et al. Transplantation with autologous bone marrow-derived mesenchymal stem cells for alcoholic cirrhosis: phase 2 trial. Hepatology 2016;64:2185 2197.

69. Xu L, Gong Y, Wang B, Shi K, Hou Y, Wang L, et al. Randomized trial of autologous bone marrow mesenchymal stem cells transplantation for hepatitis B virus cirrhosis: regulation of Treg/Th17 cells. J Gastroenterol Hepatol 2014;29:1620-1628.

70. Moroni F, Dwyer BJ, Graham C, Pass C, Bailey L, Ritchie L, et al. Safety profile of autologous macrophage therapy for liver cirrhosis. Nat Med 2019;25:1560-1565.

71. Maas SL, De Vrij J, Broekman ML. Quantification and size-profiling of extracellular vesicles using tunable resistive pulse sensing. J Vis Exp 2014;(92):e51623.

72. Gnecchi M, Danieli P, Malpasso G, Ciuffreda MC. Paracrine mechanisms of mesenchymal stem cells in tissue repair. Methods Mol Biol 2016;1416:123-146.

73. Braicu C, Tomuleasa C, Monroig P, Cucuianu A, Berindan-Neagoe I, Calin GA. Exosomes as divine messengers: are they the Hermes of modern molecular oncology? Cell Death Differ 2015;22:34-45.

74. Zhou Y, Xu H, Xu W, Wang B, Wu H, Tao Y, et al. Exosomes released by human umbilical cord mesenchymal stem cells protect against cisplatin-induced renal oxidative stress and apoptosis in vivo and in vitro. Stem Cell Res Ther 2013;4:34.

75. Arslan F, Lai RC, Smeets MB, Akeroyd L, Choo A, Aguor EN, et al. Mesenchymal stem cell-derived exosomes increase ATP levels, decrease oxidative stress and activate PI3K/Akt pathway to enhance myocardial viability and prevent adverse remodeling after myocardial ischemia/reperfusion injury. Stem Cell Res 2013;10:301-312.

76. Bian S, Zhang L, Duan L, Wang X, Min Y, Yu H. Extracellular vesicles derived from human bone marrow mesenchymal stem cells promote angiogenesis in a rat myocardial infarction model. J Mol Med (Berl) 2014;92:387-397.

77. Teng X, Chen L, Chen W, Yang J, Yang Z, Shen Z. Mesenchymal stem cell-derived exosomes improve the microenvironment of infarcted myocardium contributing to angiogenesis and anti-inflammation. Cell Physiol Biochem 2015;37:2415-2424.

78. Rager TM, Olson JK, Zhou Y, Wang Y, Besner GE. Exosomes secreted from bone marrow-derived mesenchymal stem cells protect the intestines from experimental necrotizing enterocolitis. J Pediatr Surg 2016;51:942-947.

79. Lee C, Mitsialis SA, Aslam M, Vitali SH, Vergadi E, Konstantinou G, et al. Exosomes mediate the cytoprotective action of mesenchymal stromal cells on hypoxia-induced pulmonary hypertension. Circulation 2012;126:2601-2611.

80. Aliotta JM, Pereira M, Wen S, Dooner MS, Del Tatto M, Papa E, et al. Exosomes induce and reverse monocrotaline-induced pulmonary hypertension in mice. Cardiovasc Res 2016;110:319-330.

81. Zhu YG, Feng XM, Abbott J, Fang XH, Hao Q, Monsel A, et al. Human mesenchymal stem cell microvesicles for treatment of Esch- 
erichia coli endotoxin-induced acute lung injury in mice. Stem Cells 2014:32:116-125.

82. Xin H, Li Y, Cui Y, Yang JJ, Zhang ZG, Chopp M. Systemic administration of exosomes released from mesenchymal stromal cells promote functional recovery and neurovascular plasticity after stroke in rats. J Cereb Blood Flow Metab 2013;33:1711-1715.

83. Ophelders DR, Wolfs TG, Jellema RK, Zwanenburg A, Andriessen $P$, Delhaas $T$, et al. Mesenchymal stromal cell-derived extracellular vesicles protect the fetal brain after hypoxia-ischemia. Stem Cells Transl Med 2016:5:754-763.

84. Zhang B, Wang M, Gong A, Zhang X, Wu X, Zhu Y, et al. HucMSCexosome mediated-Wnt4 signaling is required for cutaneous wound healing. Stem Cells 2015;33:2158-2168.

85. Zhang B, Wu X, Zhang X, Sun Y, Yan Y, Shi H, et al. Human umbilical cord mesenchymal stem cell exosomes enhance angiogenesis through the Wnt4/ß-catenin pathway. Stem Cells Transl Med 2015;4: 513-522.

86. Shabbir A, Cox A, Rodriguez-Menocal L, Salgado M, Van Badiavas E. Mesenchymal stem cell exosomes induce proliferation and migration of normal and chronic wound fibroblasts, and enhance angiogenesis in vitro. Stem Cells Dev 2015;24:1635-1647.

87. Blazquez R, Sanchez-Margallo FM, de la Rosa O, Dalemans W, Alvarez $V$, Tarazona $R$, et al. Immunomodulatory potential of human adipose mesenchymal stem cells derived exosomes on in vitro stimulated T cells. Front Immunol 2014;5:556.

88. Zhang B, Yin Y, Lai RC, Tan SS, Choo AB, Lim SK. Mesenchymal stem cells secrete immunologically active exosomes. Stem Cells Dev 2014;23:1233-1244.

89. Reis LA, Borges FT, Simões MJ, Borges AA, Sinigaglia-Coimbra R, Schor N. Bone marrow-derived mesenchymal stem cells repaired but did not prevent gentamicin-induced acute kidney injury through paracrine effects in rats. PLOS One 2012;7:e44092.

90. Doeppner TR, Herz J, Görgens A, Schlechter J, Ludwig AK, Radtke S, et al. Extracellular vesicles improve post-stroke neuroregeneration and prevent postischemic immunosuppression. Stem Cells Transl Med 2015;4:1131-1143.

91. Choi M, Ban T, Rhim T. Therapeutic use of stem cell transplantation for cell replacement or cytoprotective effect of microvesicle released from mesenchymal stem cell. Mol Cells 2014;37:133-139.

92. Tan CY, Lai RC, Wong W, Dan YY, Lim SK, Ho HK. Mesenchymal stem cell-derived exosomes promote hepatic regeneration in druginduced liver injury models. Stem Cell Res Ther 2014;5:76.

93. Li T, Yan Y, Wang B, Qian H, Zhang X, Shen L, et al. Exosomes derived from human umbilical cord mesenchymal stem cells alleviate liver fibrosis. Stem Cells Dev 2013;22:845-854.

94. Jiang L, Zhang S, Hu H, Yang J, Wang X, Ma Y, et al. Exosomes derived from human umbilical cord mesenchymal stem cells alleviate acute liver failure by reducing the activity of the NLRP3 inflammasome in macrophages. Biochem Biophys Res Commun 2019;508:735-741.

95. Haga H, Yan IK, Takahashi K, Matsuda A, Patel T. Extracellular vesicles from bone marrow-derived mesenchymal stem cells improve survival from lethal hepatic failure in mice. Stem Cells Transl Med 2017;6:1262-1272.

93. Povero D, Pinatel EM, Leszczynska A, Goyal NP, Nishio T, Kim J, et al. Human induced pluripotent stem cell-derived extracellular vesicles reduce hepatic stellate cell activation and liver fibrosis. $J \mathrm{Cl}$ Insight 2019;5:e125652.

97. Borrelli DA, Yankson K, Shukla N, Vilanilam G, Ticer T, Wolfram J. Extracellular vesicle therapeutics for liver disease. J Control Release 2018;273:86-98.

98. Imai T, Takahashi Y, Nishikawa M, Kato K, Morishita M, Yamashita $T$, et al. Macrophage-dependent clearance of systemically administered B16BL6-derived exosomes from the blood circulation in mice. J Extracell Vesicles 2015;4:26238.

99. Batrakova EV, Kim MS. Using exosomes, naturally-equipped nanocarriers, for drug delivery. J Control Release 2015;219:396-405. 